\title{
Critères formels pour le datif non lexical en français
}

\section{JOHAN ROORYCK}

\section{INTRODUCTION*}

Dans plusieurs articles récents, le datif non lexical a été étudié de différents points de vue. L'analyse de Barnes $(1980,1985)$ propose une explication du datif non lexical dans le cadre de la Grammaire Fonctionelle de Dik (1978). Herslund (1983) a développé une analyse transformationnelle de ce que nous considérons comme un type particulier de datif non lexical : le datif de la possession inaliénable. Enfin, Olsson (1984) a présenté une analyse descriptive et quantitative des datifs non lexicaux basée sur un corpus littéraire. Aucun de ces auteurs n'offre cependant une définition syntaxique univoque qui stipule en quoi le datif non lexical s'oppose au datif lexical. Dans cet article, nous'voulons d'abord discuter les critères syntaxiques qui permettent d'établir cette distinction. Dans le second paragraphe, nous développerons une explication pour l'acceptabilité variable des datifs non lexicaux lexicalisés, qui sert d'alternative à celle de Barnes $(1980,1985)$. Un troisième paragraphe traitera le statut du datif non lexical dans la phrase. Finalement, nous montrerons comment cette approche formelle peut rendre compte de certains cas ambigus.

Contrairement aux analyses de Barnes $(1980,1985)$ et de Herslund (1983), qui se situent dans un cadre théorique particulier, nos observations ne feront que spécifier les conditions syntaxiques descriptives qui devraient être intégrées à toute théorie syntaxique qui s'occupe de ce problème.

\section{LE DATIF LEXICAL ET LE DATIF NON LEXICAL ${ }^{1}$}

Comme le remarque Barnes (1985), la grammaire traditionnelle considère les pronoms clitiques datifs de (1) comme des substituts attachés au verbe, des éléments qui remplacent les compléments prépositionnels de type animé introduits par la préposition à. Cette correspondance est exemplifiée dans (2).

(1) me (1sg.) te (2sg.) lui (3sg.) nous (1pl.) vous (2pl.) leur (3pl.)

(2) a. Je lui donne le livre. / Je donne le livre à Marie.

b. Elle lui ressemble. / Elle ressemble à sa mère.

Les phrases où la séquence $\grave{a}-N P$ n'est pas cliticisable constitue un premier problème pour cette approche.

(3) a. Je pense à Anatole. / * Je lui pense. / Je pense à lui.

b. Tu devras l'habituer à ton père. / *Tu devras le lui habituer. / Tu devras l'habituer à lui.

Barnes (1985) se réfère à son article de 1980 où elle explique ces cas par des implications sémantiques qui s'attachent toujours au datif clitique (Barnes (1985:160)). Nous n'approfondirons pas ce problème ici. Nous voulons toutefois nous référer à une explication alternative et plus formalisée du problème des séquences $\grave{a}-N P$ non cliticisables qui a été proposée par Blanche-Benveniste et alii (1984:43-44) dans le cadre de l'approche pronominale. Ces auteurs expliquent la différence entre les séquences à-NP cliticisables et non cliticisables par une hiérarchie des restrictions de sélection du complément datif. 
Un second problème pour l'analyse traditionnelle est l'existence de clitiques datifs non lexicalisables, ou, mieux, de clitiques datifs lexicalisés qui ne sont pas entièrement grammaticaux en français.

(4) a. Je lui ai trouvé un emploi. / ? J'ai trouvé un emploi à Théophile.

b. Le gosse lui a démoli son pull. / ?? Le gosse a démoli son pull à sa mère.

c. Elle lui a tiré dans le ventre. $/ *$ Elle a tiré dans le ventre à Jean.

Barnes (1985) appelle ce dernier type de datif non lexical. Ces compléments s'opposent par là aux datifs lexicaux de (2). Elle reprend la terminologie de Leclère (1978), qui définit un verbe à datif lexical comme un verbe qui sous-catégorise un complément de type $\grave{a}-N P$ dans le lexique. Un datif non lexical est un clitique datif auprès d'un verbe qui ne souscatégorise pas pour à-NP. Ces auteurs, tout comme Herslund (1983) et Olsson (1984), envisagent toujours le clitique datif non lexical comme un substitut pour $\grave{a}-N P$. Aucun auteur ne donne un critère syntaxique précis pour distinguer ces deux types de datifs. Barnes (1980) affirme qu'il n'y a pas de critère syntaxique qui permette de distinguer entre datifs lexicaux et non lexicaux, parce que la lexicalisation du clitique datif non lexical dans (5) est parfaitement acceptable pour ses informateurs. On pourrait y ajouter que Olsson (1984: 188, 199) cite des exemples attestés de cette lexicalisation d'un datif clitique non lexical.

(5) Je leur cuis un gâteau. / Je cuis un gâteau aux enfants.

(6) a. En face, à l'est, on a trouvé à cette politique divers avantages. Le Point, 485 : 26, 3, cité par Olsson (1984: 199)

b. J'ai toujours vu à mon père une casquette, souvent à carreaux ... F. R. Bastide, La vie rêvée, p. 73, cité par Olsson (1984: 188)

Néanmoins, nous croyons que l'existence de ces exemples ne met pas en difficulté la définition provisoire du datif non lexical comme un pronom clitique datif qui peut être difficilement lexicalisé. En effet, l'analyse quantitative de Olsson (1984) semble confirmer cette définition. Bien que Olsson cite plusieurs exemples où le datif non lexical lexicalisé est parfaitement acceptable (cfr 6), ses chiffres montrent que le datif non lexical est beaucoup plus fréquent sous la forme d'un pronom que sous la forme d'un groupe prépositionnel lexicalisé. Sur un total de 685 datifs non lexicaux, seuls 89 exemples, soit presque un sur huit, sont de type lexical et non clitique. Le rapport le plus étroit est de un datif non lexical lexicalisé pour quatre datifs non lexicaux de type clitique (par exemple trouver, entendre: Olsson (1984: 192)). Ce rapport n'est pas dû au hasard, car le complément d'objet direct de ces mêmes constructions est presque toujours de type nominal (506 contre 179 exemples). Ces chiffres tendent à confirmer que les constructions où le datif non lexical est lexicalisé sont marginales, même si leur acceptabilité varie. La variation d'acceptabilité des datifs non lexicaux lexicalisés montre que ce datif de forme clitique n'a pas de rapport univoque avec une forme non clitique, lexicalisée. On ne peut donc maintenir, comme Herslund (1983), que le datif non lexical dérive d'une forme sousjacente $\grave{a}-N P$.

Le caractère fondamentalement clitique du datif non lexical, et son statut marginal s'il est lexicalisé, peut être prouvé à partir de critères purement formels, à moins de prendre en compte la relation du datif non lexical avec les autres compléments de la phrase. Les phrases dont l'acceptabilité varie si le clitique datif est lexicalisé deviennent totalement inacceptables si le complément d'objet direct est pronominalisé en même temps.

(7) a. * (Quant à un emploi,) j'en ai trouvé un à Théophile.

b. * (Ce pull,) le gosse l'a démoli à sa mère.

c. * (Quant au gâteau,) j'en ai cuit un aux enfants. 
La construction où le complément d'objet direct est pronominalisé et le complément d'objet indirect lexicalisé est parfaitement acceptable pour les datifs lexicaux des verbes transitifs.

(8) a. (Ce livre,) je le donne à Cunégonde.

b. (Ce droit,) le gouvernement l'a reconnu aux ouvriers.

c. (Ces fleurs,) elle en vend beaucoup aux touristes.

Il est intéressant de noter qu'une construction où le complément d'objet direct est relativisé ne donne pas les mêmes résultats. Nous obtenons à nouveau une variation d'acceptabilité.

(9) a. * C'est le pull que le gosse a démoli à sa mère.

b. ?? C'est la jambe qu'on a cassée à Gertrude.

c. C'est ce ton-là qu'il voulait à son roman.

Un second critère syntaxique qui permet de distinguer les datifs lexicaux et non lexicaux de façon univoque est la passivation. Ruwet (1982:180) utilise cette opération dans sa critique de la dérivation transformationnelle d'un type de phrases relaté. Toutefois, il n'établit pas la distinction qui nous intéresse $\mathrm{ici}^{2}$. La passivation des phrases qui contiennent un datif non lexical produit des phrases inacceptables.

(10) a. *? Un emploi lui a été trouvé.

* Un emploi a été trouvé à Théophile.

b. *? Ce pull lui a été démoli par le gosse.

* Ce pull a été démoli à Léontine.

c. * Un gâteau leur a été cuit.

* Un gâteau a été cuit aux gosses.

Une fois de plus, les datifs lexicaux ne possèdent pas cette caractéristique ${ }^{3}$.

(11) a. Ce livre lui a été donné.

Ce livre a été donné à Cunégonde.

b. Ce droit leur a été reconnu.

Ce droit a été reconnu aux ouvriers.

c. Beaucoup de fleurs leur ont été vendues.

Beaucoup de fleurs ont été vendues aux touristes.

Nous n'avons pas d'explication pour le contraste subtil qui existe pour nos informateurs entre certaines phrases passives avec un clitique datif et les phrases avec un datif lexicalisé de (10).

Nous pouvons donc avancer deux critères syntaxiques univoques qui permettent d'établir la distinction entre datifs lexicaux et datifs non lexicaux : d'une part, la construction qui lexicalise le datif clitique tout en pronominalisant le complément d'objet direct, et, d'autre part, la passivation.

On pourrait objecter que ces tests syntaxiques ne font que distinguer les datifs lexicaux et non lexicaux des verbes transitifs. Les critères en question ne peuvent être appliqués aux datifs des verbes intransitifs ni aux verbes qui sélectionnent un complément prépositionnel (cfr aussi (4c)).

(12) a. Cela lui appartient. / Cela appartient à Frédéric.

* Cela appartient.

b. Fabrice lui tira dessus.

* Fabrice tira dessus au cavalier prussien. 
c. Eléonore lui a tiré dans le ventre.

* Eléonore a tiré dans le ventre à Astolphe.

Toutefois, ceci n'est qu'un faux problème puisque le statut des datifs est clair dans ces phrases. Dans (12a), la lexicalisation du clitique datif est parfaitement possible. Les exemples de (5) et (6) nous ont montré que ce critère n'est pas suffisant. Toutefois, le critère de la nécessité tel qu'il est exemplifié dans (12a) prouve indépendamment le statut lexical du datif, puisque les datifs non lexicaux peuvent toujours être supprimés librement. Dans (12b), l'inacceptabilité de $\grave{a}-N P$ illustre le fait que ce datif clitique entretient un rapport régulier avec un complément prépositionnel autre que $\grave{a}-N P$ :

(13) a. Il lui tire dessus.

Il tire sur lui.

b. Elle lui tourne autour.

Elle tourne autour de lui.

La phrase (12c) montre également que la lexicalisation du clitique datif est impossible. Dans ces deux derniers cas, il s'agit donc de datifs non lexicaux. Dans le paragraphe suivant, nous essayerons d'offrir une explication alternative pour la variation observée par Barnes (1985) pour les cas de à-NP non lexical.

\section{L'ACCEPTABILITE DE $\grave{a}-N P$ NON LEXICAL}

Dans le paragraphe précédent, nous avons observé que la lexicalisation d'un clitique datif non lexical est peu acceptable si le complément d'objet direct est lexical, et entièrement inacceptable si le complément d'objet direct est pronominal. Afin de compléter cette distribution, il est intéressant de noter que les deux éléments peuvent être pronominalisés, ce qui montre leur indépendance relative.

(14) a. Je lui en ai trouvé un (, d'emplois).

b. Le gosse le lui a démoli (, son pull).

Cette distribution des datifs et des compléments d'objet direct lexicaux et pronominaux montre clairement le statut marginal de $\grave{a}-N P$ non lexical.

Néanmoins, la variation d'acceptabilité exemplifiée dans (5) et (6) doit être expliquée. Il ne semble pas possible d'expliquer cette variation à partir de critères purement formels. Nous essayerons donc d'offrir un commentaire critique de l'analyse de Barnes (1985), et de présenter une analyse alternative plus simple qui devrait rendre compte des mêmes données.

Barnes (1985) utilise l'argument de l'analogie sémantique afin de justifier l'inacceptabilité relative des phrases de (4). A son avis, les datifs lexicaux sont marqués comme des "objets seconds" du verbe. Ces compléments sont associés par là à la caractérisation sémantique de Thème. Barnes (1985) suggère que l'alternance entre $\grave{a}-N P$ et lui, ainsi que le fait que à marque la thématicité, permet l'apparition d'un complément non lexical $\grave{a}-N P$ qui est marqué pour la thématicité par analogie. Quelque vague que puisse être cette explication analogique, l'idée de l'influence d'une structure sur une autre structure qui lui est formellement apparentée ne peut être exclue a priori. Toutefois, la contrainte syntaxique formulée par Barnes (1985: 181) pour ce complément à- $N P$ " étendu " est beaucoup moins plausible. Pour expliquer l'inacceptabilité de $\grave{a}-N P$ dans (15), Barnes (1985: 182, 194) formule une condition qui exclut la possibilité de deux complément prépositionnels dans une phrase française ${ }^{4}$.

(15) a. * Les gosses ont gribouillé sur tous les murs à Marie.

b. * Elle a tiré dans le ventre à Jean. 
Il est facile de montrer que cette condition n'est pas valable en français.

(16) a. Chlöé a acheté une écharpe pour Nestor avec Melpomène.

b. Zöé a donné une rivière de perles à Aristide pour Clitandre.

La contrainte n'est même pas d'application pour les datifs non lexicaux.

(17) a. *? Les gosses ont dessiné des ânes sur tous les murs à Marie.

b. ? Les gosses ont dessiné à Marie des ànes sur tous les murs.

Pour plusieurs informateurs, la phrase (17b) est nettement meilleure que (17a), même si elle est légèrement étrange.

En plus de cette contrainte syntaxique, Barnes (1985) formule des contraintes sémantiques qui s'appliquent au complément $\grave{a}-N P$ non lexical ou "étendu ». Ainsi, elle est amenée à justifier tant l'acceptabilité relative de (182) que l'inacceptabilité de (18b) par une confusion avec la lecture adnominale de $\grave{a}-N P$, qui appartient au français populaire.

(18) a. ? / OK On a cassé le bras à Jean. (Barnes (1985: (34c) / (73))

b. ?? Je refais ces reliures à Duval. (Barnes (1985:(56))

Finalement, Barnes (1985) ne peut qu'observer que les datifs maléfactifs sont moins acceptables que les datifs bénéfactifs dans un contexte $\grave{a}-N P$ non lexical. Cette observation est une conséquence logique de l'analyse que nous présenterons ci-dessous. Nous essayerons de décrire la même variation d'acceptabilité que celle décrite par Barnes (1985), parce que les jugements d'acceptabilité qu'elle avance sont empiriquement bien fondés.

Tout d'abord, nous éliminerons les cas où la séquence à- $N P$ non lexicale est une lexicalisation d'un datif clitique qui entretient une relation de possession inaliénable avec le complément d'objet direct ${ }^{5}$. Cette relation inaliénable concerne normalement des parties du corps ou des vêtements (Herslund (1983), Olsson (1984: 198)). Le fait que ces phrases sont acceptables peut être attribué à cette relation sémantique. La phrase (20b) montre que la lecture adnominale ne peut être invoquée pour expliquer l'acceptabilité des datifs non lexicaux de la possession inaliénable comme le fait Barnes (1985) ${ }^{6}$. Dans (20a), la relation de possession inaliénable est présente sous la forme d'une relation du tout à la partie entre le datif et le complément d'objet direct.

(19) a. (?) On a tordu le poignet à Pierre.

b. (?) On a tiré les oreilles à Marc.

(20) a. En face, à l'Est, on a trouvé à cette politique divers avantages. Le Point, 485 : 26, 3, cité par Olsson (1984: 199)

b. J'ai toujours vu à mon père une casquette, souvent à carreaux . . . F. R. Bastide, La vie rêvée, p. 73, cité par Olsson (1984: 188))

c. ... ce regard d'affection que je n'ai jamais connu à personne d'autre (. . ) C. Albaret, Monsieur Proust, p. 449-450, cité par Olsson (1984: 197)

d. Les hommes trouvaient des grâces de toutes sortes aux jeunes filles au pair. Le Point, 397:92,2 cité par Olsson (1984: 198)

Les autres cas du datif non lexical lexicalisé peuvent être expliquées par un autre type d'analogie que celui développé par Barnes (1985). Dans la plupart des cas, l'acceptabilité de la séquence à- $N P$ de ces phrases est parallèle à l'acceptabilité de pour-NP dans les mêmes phrases. L'acceptabilité de ces phrases est alors une conséquence de la polysémie de $\grave{a}$, préposition qui permet des emplois proches de pour.

(21) a. ? Paul a cassé ces trois verres à Marie/ ?? pour Marie.

b. ? Elle a démoli son pull à sa mère/ ?? pour sa mère. 
(22) a. ?? Paul a fait une bronchite à sa mère/ pour sa mère.

b. * Paul a attrapé une bronchite à/ pour sa mère.

c. * Paul a bu trois pastis à/ pour sa mère sans dire merci.

Les phrases suivantes sont toutes légèrement étranges aux yeux des informateurs de Barnes (1985). Il n'est pas surprenant de constater que la version avec pour est parfaitement acceptable.

(23) a. Elle tricote un pull (?) au bébé/ pour le bébé.

b. Il choisit des bagues (?) à sa femme/ pour sa femme.

c. Paul a joué le cinq (?) à Marie/ pour Marie dans la dernière course.

Toutefois, certains cas semblent falsifier l'observation que l'acceptabilité de à- $N P$ est parallèle à celle de pour-NP. La préposition pour est acceptable dans les phrases suivantes, mais la séquence $\grave{a}-N P$ est exclue.

(24) a. ?? J'ai rajeuni ces/des plantes à Paul/ OK pour Paul.

b. ?? Je refais ces reliures à Duval/ OK pour Duval.

Nous sommes d'accord avec Barnes (1985:185) qui attribue l'inacceptabilité à une confusion avec la lecture adnominale de $\grave{a}$-NP. Il faut avouer que cette confusion avec la lecture adnominale n'est pas toujours claire : des facteurs sémantiques semblent influencer les jugements d'acceptabilité. Barnes (1985) n'offre pas d'explication pour la différence d'acceptabilité des exemples suivants.

(25) a. ? J'ai corrigé ces devoirs à Jean. (Barnes (1985: (52)))

b. ?? J'ai corrigé ces épreuves à Jean. (Barnes (1985: (55)))

Une explication possible pour cette opposition pourrait être trouvée dans l'observation que la lecture adnominale s'obtient plus facilement dans (25b), parce que le contexte sémantique suggère que les épreuves ont également été produites par Jean. Par conséquent, une confusion comme dans (24) est possible. Par contre, dans (25a), les devoirs sont assimilés sémantiquement à une chose externe qui est imposée à Jean. Le lien possessif possible est plus évident dans (25b) que dans (25a). Il y a donc une confusion entre une lecture adnominale et la lecture où la séquence $\grave{a}-N P$ est un complément indépendant. Le même raisonnement peut expliquer pourquoi les phrases de (21) sont meilleures. La quantification du complément d'objet direct (trois) dans (21a) et la présence de deux marqueurs de la possession dans (21b), rendent la lecture adnominale de $\grave{a}-N P$ plutôt difficile. Une lecture où la séquence $\grave{a}-N P$ est indépendante avec un sens proche de pour$N P$ est la seule interprétation possible. Notons que cette analyse donne également une explication pour l'opposition dans (17): malgré la quantification du complément prépositionnel, le contexte sémantique induit une lecture adnominale, possessive de $\grave{a}-N P$ dans (17a). La position de $\grave{a}-N P$ dans (17b) exclut cette lecture, et la phrase est presque acceptable.

Un autre problème pour cette analyse se trouve dans la phrase suivante, où pour-NP est inacceptable.

(26) Le capitaine trouvait à l'alchimiste l'air soucieux et fatigué (M. Yourcenar, L'ceuvre au noir, p. 137)

Toutefois, cette phrase peut être expliqué par le caractère idiomatique de trouver l'air Adj. On peut observer que la focalisation n'est pas possible pour le complément d'objet direct de (26), ce qui est bel et bien le cas pour le complément d'objet direct des phrases de (4).

(27) * C'est l'air soucieux et fatigué que le capitaine trouvait à l'alchimiste. 
(28) a. C'est un emploi que je lui ai trouvé.

b. ? C'est un emploi que j'ai trouvé à Théophile.

Cet argument n'est toutefois pas suffisant, puisque les phrases du type (4) manifestent une variation d'acceptabilité pour $\grave{a}-N P$ non lexical. Néanmoins, on constate que la pronominalisation ou la passivation du complément d'objet direct ne sont pas possibles pour (26). Cela prouve de façon convaincante le statut idiomatique du complément nominal postverbal l'air. En effet, des expressions comme avoir peur, foutre le camp ne permettent pas non plus la reformulation du complément nominal fixe qui fait partie du verbe ${ }^{7}$.

(29) * Le capitaine le lui trouvait (l'air soucieux).

* L'air (lui) avait été trouvé.

(30) a. Il a peur./* Il l'a. / * C'est peur qu'il a.

b. Il fout le camp. ${ }^{*}$ Il le fout./* Le camp a été foutu.

Notre analyse semble donc capable d'établir la distinction importante entre les séquences $\grave{a}-N P$ non lexicales acceptables et inacceptables. D’autres facteurs sémantiques jouent probablement un rôle dans l'acceptabilité de ces phrases. Un test plus large auprès de plusieurs informateurs devrait montrer si ces différences sont réellement pertinentes. La distribution des clitiques non lexicaux et $\grave{a}-N P$ montre clairement que la construction $\grave{a}-N P$ n'est que marginalement acceptable. Les conditions qui gouvernent la variation de cette acceptabilité peuvent être formulées d'une façon simple et précise. Dans le paragraphe suivant, nous essayerons de définir le statut du clitique datif non lexical dans la phrase.

\section{LE STATUT SYNTAXIQUE DU DATIF NON LEXICAL}

Le caractère marginal de à-NP non lexical justifie l'analyse du datif clitique non lexical comme un morphème optionnel attaché au verbe. Dans les termes de Blanche-Benveniste et alii (1984), on pourrait dire qu'il n'y a pas de «proportionnalité » entre le clitique non lexical et $\grave{a}-N P$ non lexical. Blanche-Benveniste et alii (1984:72) notent que ce clitique datif entretient une relation de "solidarité " avec la fonction du complément d'objet direct. Comme Herslund (1983), ils n'examinent que des cas de possession inaliénable (cfr note 5). Les auteurs affirment que l'impossibilité de (31) suffit pour prouver cette solidarité avec la fonction du complément d'objet direct.

(31) a. Je lui casse la jambe.

b. * Je lui casse.

Toutefois, il semble que ce test est une condition nécessaire mais non suffisante pour établir un lien de solidarité sélectionnelle entre le clitique datif non lexical et la fonction du complément d'objet direct. En effet, pour certains datifs lexicaux, la suppression du complément d'objet direct donne également un résultat inacceptable. Pourtant, dans ces cas-si, nous ne voudrions pas parler d'une solidarité sélectionnelle dans la mesure où le datif lexical et le complément d'objet direct exercent des fonctions syntaxiques indépendantes auprès du verbe constructeur.

(32) a. Ludwig consacra une symphonie à un général corse.

b. * Ludwig lui consacra.

Le critère de la passivation que nous avons utilisé dans le premier paragraphe est un argument suffisant pour prouver cette solidarité entre le clitique datif non lexical et la fonction du complément d'objet direct. Si le clitique datif était uniquement sélectionné par 
le verbe, il devrait ètre possible de l'exprimer dans la construction passive de ce même verbe. Les exemples (10) et (33) montrent que cela n'est pas le cas. Nous pouvons donc conclure que cette solidarité sélectionnelle existe vraiment, et que la fonction du complément d'objet direct contient un élément optionnel qui est discontinu et exprimé sur le verbe comme un datif clitique.

(33) * La jambe lui a été cassée.

Le fait que le datif clitique non lexical constitue une unité sélectionnelle avec le complément d'objet direct, permet d'expliquer pourquoi, en français standard, le déterminant du complément d'objet direct est toujours défini si ce complément entretient une relation de possession inaliénable avec le référent du datif non lexical.

(34) Je lui ai cassé la/*sa jambe.

En effet, il est impossible d'exprimer ce type de possession deux fois dans le même complément.

(35) * J'ai cassé sa $\mathrm{a}_{\mathrm{i}}$ jambe de $\mathrm{Jean}_{\mathrm{i}}$

Nous croyons que c'est essentiellement ce résultat qui est visé par Herslund (1983) quand il essaie de dériver le datif de la possession inaliénable d'un complément adnominal ${ }^{8}$. Pour les verbes intransitifs qui possèdent un datif clitique non lexical, la solidarité est établie avec un complément prépositionnel. L'inacceptabilité de ces phrases sans complément prépositionnel est un argument à la fois nécessaire et suffisant pour la solidarité entre ces deux éléments, car des verbes qui sous-catégoriseraient et un datif lexical et un complément prépositionnel de type locatif qui ne peut être supprimé, n'existent pas en français.

(36) a. Les enfants lui ont gribouillé sur tous les murs.

b. * Les enfants lui ont gribouillé.

(37) a. Fabrice lui tira dessus.

b. Elle lui tira dans le ventre.

c. * Fabrice/ elle lui tira.

Afin de déterminer la valeur exacte de cette notion de solidarité, il est intéressant de comparer le clitique datif non lexical à d'autres clitiques qui manifestent les mêmes caractéristiques. Blanche-Benveniste et alii (1984) analysent le clitique datif non lexical de la même manière que les clitiques en exemplifiés dans (38). Dans leur analyse, le clitique datif non lexical appartient à la valence du verbe, le clitique en de (38a) à la valence de l'adjectif, et le clitique en de (38b) à la valence d'un certain type sémantique de compléments nominaux.

(38) a. J'en suis fier.

b. J'en connais le prix/ une partie.

Toutefois, il y a une différence cruciale entre le clitique en dans (38) et le datif clitique non lexical. Le complément adnominal ou adjectival et le NP ou AP ne peuvent être pronominalisés en même temps, parce que deux pronoms clitiques ne peuvent avoir une relation de dépendance en français.

(39) a.* Je l'en suis.

b. * Je l'en connais.

Par contre, comme nous l'avons montré dans (14), le datif non lexical et le complément d'objet direct dont il est solidaire peuvent être pronominalisés à la fois. Cette différence 
semble montrer que deux types de solidarité doivent être distingués. Dans (38), la relation de dépendance entre le clitique et NP ou AP est telle que le clitique en est subordonné au NP ou AP qui le sélectionne. Dans ce cas, on peut réellement parler de compléments discontinus. Le lien entre le datif non lexical et un complément sous-catégorisé par le verbe (complément d'objet direct ou prépositionnel) est une relation de solidarité mais non de dépendance sélectionnelle.

\section{QUELQUES CAS D'AMBIGUITE}

Il est bien connu que les datifs clitiques de certains verbes en français sont ambigus entre une interprétation comme Bénéficiaire ou comme Source.

(40) a. Je lui ai acheté cette voiture.

b. Je lui ai pris ce disque.

L'analyse présentée ici fournit une explication formelle pour cette ambiguité interprétative. Quand on applique les critères développés dans le premier paragraphe aux phrases de (40), seule l'interpretation du complément d'objet indirect comme source subsiste.

(41) a. Je l'ai acheté à mon frère.

b. Je l'ai pris à Donatienne.

(42) a. Cette voiture lui a été achetée par Guillaume.

b. Ce disque lui a été pris hier soir.

Comme seul le datif lexical peut occuper plusieurs positions dans la phrase, nous pouvons conclure que le datif interprété comme source est un datif lexical (lui---de lui; $\mathrm{P}_{3}$ dans la terminologie de Blanche-Benveniste et alii (1984)). Selon les critères que nous avons développés plus haut, un datif non lexical ne peut apparaître que dans une construction active comme $(40)^{9}$. Par conséquent, on peut dire que des verbes comme acheter, prendre sont caractérisés par la sélection d'un datif lexical (Source) et d'un datif non lexical (Bénéficiaire). Dans la construction de (40), cette différence structurale est neutralisée, ce qui provoque une ambiguïté interprétative.

\section{CONCLUSION}

La possibilité pour un datif de figurer dans plusieurs constructions reformulées de la phrase semble fournir un moyen intéressant pour distinguer les datifs lexicaux et non lexicaux. Un datif lexical, sous-catégorisé par le verbe, se maintient dans toutes les reformulations imposées à la phrase construite par ce verbe. Un datif non lexical n'est parfaitement acceptable que quand il est exprimé comme un clitique sur le verbe. Ce clitique datif n'a pas le statut d'un complément sous-catégorisé par le verbe : un lien de solidarité sélectionnelle en fait un élément lié au complément d'objet direct ou prépositionnel sélectionné par le verbe. L'acceptabilité variable des datifs non lexicaux lexicalisés peut être expliquée par l'interaction de facteurs syntaxiques et sémantiques. Enfin, cette analyse permet d'établir une distinction formelle dans un cas intéressant d'ambiguité interprétative.

Departement Linguïstiek

Faculteit van de Letteren en de Wijsbegeerte

Blijde-Inkomststraat 21

B-3000 Leuven 


\section{NOTES}

* Je voudrais remercier Ludo Melis, Karel Van den Eynde et Serge Verlinde pour leurs suggestions, bien qu'ils ne soient pas nécessairement d'accord avec ce que j'ai écrit ici. Ma gratitude s'adresse également au Fonds National de Recherche Scientifique (Belgique) pour son soutien financier.

1 Dans cet article, nous nous ne parlerons pas du « datif éthique ». Même si l'on peut considérer ce datif éthique comme un datif clitique qui n'est pas lexicalisable, il doit être distingué du datif non lexical. Contrairement au datif non lexical, le datif éthique est limité à la première et la seconde personne, et il peut être redoublé. En plus, il peut se trouver dans la même phrase avec un datif non lexical.

Avez vous vu comme je te vous lui ai craché à la figure? (Victor Hugo, Les misérables, I,5, 13 cité par Grevisse (1980 : par 1063))

Notons encore que nous ne croyons pas que le datif non lexical soit limité aux verbes «agentifs » ou " non statifs " (Donaldson (1973)) ou aux verbes de «phase 2 » (Blanche-Benveniste (1984: 74). Comme le montrent les exemples suivants, cette contrainte ne concerne qu'un sous-ensemble des datifs non lexicaux, les datifs de la possession inalienable.

Jamais je ne lui ai entendu un propos mesquin (F. Marceau, Chair et cuir, p. 208, cité par Olsson (1984: 193)).

Je lui ai vu une jupe bizarre.

* Je ne lui ai jamais entendu la voix.

* Je ne lui ai jamais vu le mollet.

2 Ruwet (1982:172) distingue un "datif épistémique " pour certains verbes qui peuvent avoir des compléments phrastiques et une construction avec un complément d'objet direct et un datif.

a. Je lui croyais une maîtresse dans chaque port.

Je croyais qu'il avait une maîtresse dans chaque port.

b. Le gouvernement a reconnu le droit de grève aux ouvriers.

Le gouvernement a reconnu que les ouvriers avaient le droit de grève.

La notion de datif épistémique couvre un sous-ensemble des datifs lexicaux et un sous-ensemble des datifs non lexicaux. Dans (a), le clitique datif ne peut être lexicalisé et il ne peut pas subsister après la passivation. Dans (b), la lexicalisation et la pronominalisation sont parfaitement possibles, et le datif subsiste après la passivation. Le datif de (a) est non lexical, celui de (b) est lexical. Contrairement à Olsson (1983), nous croyons donc que le verbe reconnaître a un datif lexical.

3 La passivation semble également distinguer les datifs lexicaux et non lexicaux en néerlandais (K. Van den Eynde, communication personnelle)

Ik heb hem dat boek gegeven./ Dat boek werd hem gegeven.

Ik heb hem dat boek gekocht./* Dat boek werd hem gekocht.

4 Barnes (1985: 194 note 23) stipule cette condition de la façon suivante: "I.e. VP $--\rightarrow$ V-(NP)(PP), as opposed to S --> NP-VP-(PP)*, where * indicates the possibility of more than one occurrence of PP. » Notons encore que certains exemples de l'auteur contredisent également cette condition étrange (cfr Barnes (1985:(22d))).

5 Comme le note Barnes (1985) les datifs de la possession inaliénable constituent un sous-ensemble des datifs non lexicaux en général. Les critères développés dans le paragraphe précédent peuvent suffir pour illustrer ce point.

* Le poignet lui a été tordu.

* On l'a tordu à Pierre.

Herslund (1983) a essayé de distinguer ces datifs des datifs non lexicaux "étendus ». Cet auteur affirme qu'il y a une correspondance formelle entre le datif non lexical «étendu » et le complément prépositionnel introduit par pour. Il est vrai que cette correspondance ne se retrouve pas pour les datifs de la possession inaliénable. Toutefois, nous montrerons plus loin que la préposition pour ne se substitue pas non plus à certains datifs non lexicaux qui n'impliquent pourtant pas une relation de possession inalienable ou du tout à la partie. Le critère formel proposé par Herslund (1983) pour distinguer entre datifs de possession inaliénable et datifs étendus ne peut donc être généralisé.

$6 \mathrm{La}$ lecture adnominale n'est pas non plus possible pour les phrases de (19). Si elle était possible, la focalisation de l'ensemble $N P$ à $N P$ devrait être acceptable, ce qui n'est pas le cas.

* C'est le poignet à Pierre qu'on a tordu.

* Ce sont les oreilles à Jacques qu'on a tirées.

7 Olsson (1983) utilise la construction Je lui trouve mauvaise mine comme un exemple type du datif non lexical. Il analyse mauvaise mine comme le complément d'objet direct de trouver. L'impossibilité de reformuler ce complément nominal montre le statut idiomatique de trouver Adj mine. La même chose vaut pour trouver l'air Adj. 
$8 \mathrm{Il}$ est inutile de préciser que nous n'acceptons pas pareille dérivation qui ne permettrait pas d'expliquer les deux tests permettant d'identifier le datif non lexical. Dans un cadre transformationnel, le datif non lexical devrait être généré dans la base comme élément discontinu du NP postverbal.

9 Cette affirmation est restreinte aux cas où le clitique datif non lexical est lié à un complément d'objet direct. Le datif non lexical est évidemment possible dans une structure passive s'il est lié à un complément prépositionnel.

* Ce costume lui a été taillé dans les règles de l'art.

Ce costume lui a été taillé sur mesure.

Dans la première phrase, le clitique datif non lexical ne peut être lié au complément prépositionnel, puisque ce complément n'est pas sous-catégorisé par le verbe. Dans la seconde phrase, et dans la phrase suivante, le complément prépositionnel est bel et bien sous-catégorisé par le verbe.

Une balle lui a été tirée dans le ventre.

\section{BIBLIOGRAPHIE}

Barnes, Betsy. 1980. "The notion of «Dative » in Linguistic Theory and the Grammar of French. » Linguisticae Investigationes IV, 245-292.

Barnes, Betsy. 1985. "A Functional Explanation of French Nonlexical Datives. "Studies in Language 9 (2), 159-195.

Blanche-Benveniste, Claire, Deulofeu, J., Stefanini, J. and Van den Eynde, K. 1984. Pronom et syntaxe. Paris : SELAF.

Dik, Simon, 1978, Functional Grammar. Amsterdam : North-Holland.

Donaldson, Weber. 1973. French Reflexive Verbs. The Hague : Mouton.

Grevisse, Maurice. 1980 ${ }^{11}$. Le bon usage. Gembloux : Duculot.

Herslund, Michael. 1983. "Le datif de la possession inaliénable en français. » In : Herslund, Michael et alii (éds.) Analyses grammaticales du français, Revue Romane numéro spécial 24, pp. 99-116.

Leclère, Christian. 1976. « Datifs syntaxiques et datif éthique. » In: Chevalier, J.-C. \& Gross, M. (éds.). Méthodes en grammaire française. Paris : Klincksieck.

Leclère, Christian. 1978. "Sur une classe de verbes datifs. "Langue française 39, 66-75.

Olsson, Hugo. 1984. « La construction Je lui trouve mauvaise mine en français moderne. » Studia Neophilologica 56: 183-213.

Ruwet, Nicolas. 1982. "Le datif épistémique en français et la condition d'opacité de Chomsky. » In : Grammaire des insultes, 172-204. Paris : Le Seuil. 